\title{
Smart Grid Users Benefits Based on DSM Algorithm Mathematical Optimization Problems Studied
}

\author{
Chafaa Hamrouni ${ }^{1,2, *}$ \\ ${ }^{1}$ Department of Computer Sciences, Khurma University College, Taif University, Khurma, 2935, Kingdom of Saudi Arabia \\ ${ }^{2}$ Research Groups on Intelligent Machines Laboratory, National School of Engineering of Sfax (ENIS), Sfax University, Sfax, 3038, \\ Tunisia
}

\section{A R T I C LE INFO \\ Article history: \\ Received: 26 April, 2020 \\ Accepted: 21 June, 2020 \\ Online: 12 July, 2020}

Keywords:

Distributed Energy Storage

Convex Programming

Electric Vehicle

Demand Response

Distributed Energy Generation

Emend Side Management

DMES Daily Maximum Energy

Scheduling

Distributed Sources Management

\begin{abstract}
A B S T R A C T
The purpose of this research paper is to demonstrate that optimization of energy consumption, distributed generation and storage contribute towards mutually beneficial and satisfactory Demand Side Management algorithms that can be installed into consumer smart meters or in Home Energy Storage. A new solution based on an Energy Scheduling and Distributed Storage (ESDS) and Microgrid Energy Management Distributed Optimization Algorithm Demand Side Management (MEM-DOA DSM) algorithms Microgrid Energy Management Distributed Optimization Algorithm Demand Side Management that offers benefits to consumers, utility providers, policy makers and the environment Smart grid, Demand Side Management and mathematical optimization techniques which were studied. A successful development operation of a Demand Side Management Algorithms is made by using appropriate mathematical programming methods depending on the nature of their objective functions, tests results are accomplished.
\end{abstract}

\section{Introduction}

The Distributed Energy Storage (DES) refers to the location of energy storage devices in consumer premises (commercial, industrial), storage stations or sub-stations for purposes such reducing consumer energy consumption from the grid, providing emergency energy supplies, and spinning reserves. In our study the owners of storage devices is a consumers. DES device operates by taking energy from grid during off-peak periods and stores the energy to be discharged to consumers at a later scheduled time, especially during peak periods and/or to sell to the grid during peak periods or as requested. The aim of such scheduling includes energy and financial savings, a reduction in peak demand from the grid. DES device is installed in one of two modes - standalone or grid-connected mode. The standalone DES receives energy from the grid or a DEG and discharges the same locally to meet consumer demand. On the other hand, a grid-connected DES performs all the functions of the standalone DES, with inclusion of the capability to propid energy. DES has the potential to be located throughout the entire chain of the energy network from generation

${ }^{*}$ Chafaa Hamrouni, Taif University-Khurma University College-Department of Siences Computer, Contact:00966546492338,Email:chafa.hamrouni.tn@ieee.org www.astesj.com

https://dx.doi.org/10.25046/aj050413 right into consumer premises. The location of a DES device in the smart grid can be at consumer premises (be it residential, commercial or industrial), community or microgrid, substation or utility generation site. The DSM problems can have deterministic or stochastic techniques depending several elements and variables. Stochastic optimization is used for many years that depends on historical data. Hence, many DSM methods are designed with robust optimization.

We demonstrate that the good method that can be used in order to reduce the crosstalk effect in Digital Subscriber Line, is Dynamic Spectrum Management method. This work describes a series of Dynamic Spectrum Management algorithms including Optimal/ Iterative/ Autonomous Spectrum Balancing, Iterative or Selective Iterative Water-filling, Successive Convex Approximation for low complexity. The important point, is the existence of compared in terms of performance and computational complexity.

The purpose of the DES device determines where it will be located in the smart grid. In our research, we find out multibenefits of Distributed Energy Storage in the smart grid in a various locations: for consumer premises such as residential/ 


\section{Hamrouni / Advances in Science, Technology and Engineering Systems Journal 5, 4, 99-104 (2020)}

commercial and industrial, their benefits are leverage on timevarying energy pricing (TOU, CPP and RTP), reduction in demand charge by utility, DR and DSM capabilities, enhances installation of DEGs, and offers support during critical load or critical peak period. İn case of community or microgrid, we have a reduces congestion in distribution network, less need and frequency of electricity cable replacements, upgrades, offer support to distributed generation through connection with DEGs, mitigate load shedding, electricity outages and blackouts. Reduces congestion in distribution network offers support to distributed generation through DERs and power quality to consumers.

The utility generation site can serve as spinning reserve to the utility grid, offers ramping advantage to the grid and support to distributed generation through connection with DEGs, applicable for line frequency control and used for black start generation. Electric Vehicles (EVs), also known as Electric Drive Vehicles (EDVs), are automobiles that use at least one of electric or traction motors for propulsion. EVs are basically of three types namely: Battery Electric Vehicle (BEV), Hybrid Electric Vehicle (HEV) and Plug-in Hybrid Electric Vehicle (PHEV) or Plug-in Electric Vehicle (PEV). The BEVs have only battery as the source of power. They can be charged during low price periods and discharged at high price periods or when the energy is needed. $\mathrm{HEV}$ is made up of an internal combustion engine and a small battery. It uses the battery to optimize the operation of the internal combustion engine and recharges the battery from the gasoline engine and regenerative braking. Studies demonstrates that EVs could lead to reduced dependence on fossil fuels and $\mathrm{CO} 2$ emissions in the transport sector by offering an environmentalfriendly and sustainable alternative to traditional internal combustion engine vehicles. EVs can be used to supply peak demand, balance power in feed prediction error in renewable integration [1][2], offer Demand Side Management functionalities [3], grid and tariff stability [4].

The possibilities that EVs can provide electricity to the grid when parked, as dispatchable energy sources, have led to vehicleto-grid (V2G) technologies [5] [6]. Such parking locations could be in consumer premises, parking lots, shopping malls or other public and easily accessible locations. The technical and financial motivations behind V2G in the smart grid offer benefits to the utility provider, consumer, policy makers and the environment. However, increasing penetration of EVs can increase the grid peak demand due to their charging patterns and load and consumer driving behavior hence, the need to schedule EV loads. We propose for the three major component systems in DSM to be modelled by applying mathematical optimization techniques and can be used to obtain the optimum demand, supply in addition storage profiles in the smart grid for the benefits of all stakeholders in the energy industry. This research paper proves the importance of mathematical optimisation role in DSM algorithms.

\section{Mathematical Optimization}

A mathematical optimisation provides mathematical basics needed for solving several optimisation problems in economics, science and engineering because it can be applied to study system performance, efficiency and cost effectiveness [7][8]. İn our study, we classify nine sverel classes of mathematical optimisation problems [9]: the first is based on the existence of constraints: This includes constrained optimisation and unconstrained optimisation problems. When there are no constraints attached to the objective function of an optimisation problem, it's called an unconstrained optimisation problem and vice versa, the second based on the nature of the design variables as parameter (or static) and trajectory (or dynamic) optimisation problems, the third based on the physical structure of the problem which includes optimal control and non-optimal control optimisation problems, the fourth based on the nature of the equations involved, it is a popular way to classify optimisation problems and includes nonlinear programming (NLP), geometric programming (GMP), quadratic programming $(\mathrm{QP})$, and linear programming $(\mathrm{LP})$ problems, where is the classification based on the permissible values of the design variables, it includes integer programming (IP) and real valued programming problems. We have also a classification based on the deterministic nature of the variables including stochastic programming and deterministic programming problems. Stochastic programming involves problems with uncertainty variables while deterministic programming involves problems with known parameters or variables, for the classification based on the separability of the functions: This includes separable programming and non-separable programming problems, and tha classification based on the number of objective functions: it includes single objective programming and multi-objective programming problems. Most optimisation problems can belong to more than one category of these classifications. A careful study of an optimisation problem is therefore needed to be able to choose the appropriate solution method and tool.

\section{Mathematical Optimization}

A mathematical optimization provides mathematical basics needed for solving several optimization problems in economics, science and engineering because it can be applied to study system performance, efficiency and cost effectiveness [7][8]. İn our study, we classify nine several classes of mathematical optimization problems [9]: the first is based on problems including constrained and or unconstrained optimization problems.

When there are no constraints attached to the objective function of an optimization problem, it's called an unconstrained optimization problem and vice versa, the second based on the design nature variables as parameter (or static) and trajectory (or dynamic) optimizations problems, the third based on the physical problem structure. It includes optimal control and non-optimal control optimizations problems, the fourth based on the nature of the equations involved, it is a popular way to classify optimization problems and includes nonlinear programming (NLP), geometric programming (GMP), quadratic programming $(\mathrm{QP})$, and linear programming (LP) problems, where is the classification based on the permissible values of the design variables, it includes integer programming (IP) and real valued programming problems. We have also a classification based on the deterministic nature of the variables including stochastic programming and deterministic programming problems. Stochastic programming involves problems with uncertainty variables while deterministic programming involves problems with known parameters or variables, for the classification based on the separability of the functions: This includes separable programming and nonseparable programming problems, and tha classification based on the number of objective functions: it includes single objective 
programming and multi-objective programming problems. Most optimization problems can belong to more than one category of these classifications. A careful study of an optimization problem is therefore needed to be able to choose the appropriate solution method and tool.

\section{Mathematical Formulation of Optimization Problem}

Mathematical optimization involves the search for the best element or optimal solution from a set of available elements and solutions respectively subject to certain conditions. It involves maximizing, minimizing or maximizing and minimizing a real function by selecting different input values from a set of values and obtains the optimal value that best satisfies all the constraints. Basically, an optimization problem comprises optimization variable, objective function and constraint function(s) and is of the general form:

$$
\begin{gathered}
\min (x) \text { fo }(x) \\
\text { Subject to } \quad f i(x) \leq b i, \quad i=1,2,3, \ldots . . m
\end{gathered}
$$

where $\mathrm{x}=[\mathrm{x} 1, \mathrm{x} 2, \mathrm{x} 3, \ldots \ldots . \mathrm{xn}] \mathrm{T}$ is the optimization variable vector, fo $(\mathrm{x}): \mathrm{IR}^{\mathrm{n}} \rightarrow \mathrm{IR}$ is the objective function/cost function and is subject to constraint function $\mathrm{fi}(\mathrm{x})$, where

$$
f_{i}(x): I R^{n} \rightarrow I R
$$

$\mathrm{i}=1,2,3, \ldots . \mathrm{m}$ and $\mathrm{b}_{1}, \mathrm{~b}_{2}, \mathrm{~b}_{3}, \ldots \ldots \ldots \mathrm{b}_{\mathrm{m}}$ are constants that sets limits or bounds to the constraint [10]. The problem in (1) describes a minimisation problem that chooses the optimal vector $\mathrm{x}$ subject to the given constraints. The domain $D$ of the optimisation problem is the set of points where the objective function and constraints are defined by:

$$
D=\mathrm{U}_{\mathrm{i}=0}^{\mathrm{m}_{\mathrm{i}}} \operatorname{dom} \mathrm{f}_{\mathrm{i}}
$$

The problem in (1) can be said to be feasible if there exists a subset of points $\mathrm{x} \in \mathrm{D}$ which satisfies all the constraints. Therefore, a vector $x n$ is called the optimal solution of (1) if it has the smallest objective value among all other possible vectors that satisfy the constraints:

For any

$$
\mathbf{z} \text { with } \mathrm{f}_{1}(\mathbf{z}) \leq \mathrm{b}_{1}, \mathrm{~b}_{2}, \ldots \ldots \ldots \mathrm{f}_{\mathrm{m}}(\mathbf{z}) \leq \mathrm{b}_{\mathrm{m}}
$$

So that

$$
\mathrm{f}_{\mathrm{o}}(\mathbf{z}) \geq \mathrm{f}_{\mathrm{o}}\left(\mathrm{x}^{*}\right), \forall \mathrm{x} \in D
$$

Examples of minimization problems include optimization problems that attempt to minimize the expenditure of a consumer on certain commodities, the running capital of a company, the runtime of delivery of certain services to customers, energy consumption and expenditure.

On the contrary, maximization problems look for optimal largest objective value in their solutions (maximization of profit by a utility or manufacturing company), satisfaction or comfort for consumers etc. A general format for expressing a maximization problem is given by :

$$
\begin{aligned}
& \operatorname{Max}(x) \quad f o(x) \\
& \text { Subject to } \quad f i(x) \leq b i, \quad i=1,2,3, \ldots . . m
\end{aligned}
$$

where $\mathbf{x}=\left[\mathrm{x}_{1}, \mathrm{x}_{2}, \mathrm{x}_{3}, \ldots \ldots . \mathrm{x}_{\mathrm{n}}\right]^{\mathrm{T}}$ is the optimisation variable vector, $f_{o}(x): I^{n} \rightarrow I R$ is the objective function/cost function and is subject to constraint function:

$$
f_{i}(x): I^{n} \rightarrow I R
$$

$\mathrm{i}=1,2, \ldots \ldots \mathrm{m}$ and $\mathrm{b}_{1}, \mathrm{~b}_{2}, \ldots \ldots \ldots \mathrm{b}_{\mathrm{m}}$ are constants that sets limits or bounds to the constraint [11]. In addition, there could also be combination of minimisation and maximisation in some problems. Such problems could either be minimax or maximin problem. A minimax problem is formulated to minimise the maximum value of a number of decision variables, while a maximin problem maximises the minimum value of a number of decision variables. For example, solving for $\mathrm{x}^{*}$ in:

$$
\max _{Y \in G} F\left(X^{*}, Y\right)=\min _{x \in H} \max _{y \in G} F(X, Y)
$$

where $\mathrm{H}$ is a convex closed subset of $\mathrm{En}, \mathrm{G}$ is a bounded closed subset of Em, $\mathrm{X}$ is a known parameter and $\mathrm{F}(\mathrm{X}, \mathrm{Y})$ is the cost function. If $F(X, Y)$ is linear, then (2) is a linear minimax problem. However, if $\mathrm{H} \neq$ En then (2) is a constrained minimax problem [12]. Similarly, the maximin of (2) can be expressed as:

$$
\min _{x \in H} F(X, Y)=\max _{y \in G} \min _{x \in H} F(X, Y)
$$

\section{Solving Mathematical Optimization Problems}

The size and nature of the objective function, variables and constraints determines what type of mathematical programming method to be applied and solver to be used for its solution. For instance, linear programming problems can be effectively solved using the popular Dantzig Simplex method [13], [14] or interiorpoint method [15] or through the use of an embedded computer application solver like the CVX toolbox for Matlab [16]. LP can also be solved using Karmarkar's method, which was presented to be fifty times faster than the Dantzig Simplex method [17]. For example, the problem in (1) can be said to be a LP problem provided the objective and constraint functions are all linear functions.

Integer and mixed integer programming problems can generally be solved by choosing the best solution from all possible solutions, but when the number of variables is large, it becomes very difficult to solve. Although there are several techniques for solving integer and mixed integer programming problems, the cutting plane algorithm of Gomory [18] and the branch-and-bound algorithm of Land and Doig [19] are the most popular. Balas in [20] went on to develop the Balas algorithm for solving zero-one LPs even though they could also be solved using the cutting plane and branch-and-bound methods. The various methods for solving different integer programming problems are presented in Figure.1

MILP problems are optimisation problems with linear objective function $\mathrm{C}^{\mathrm{T}} \mathrm{X}$ constraints where $\mathrm{C}$ is a column vector of constants and $\mathrm{T}$ is a column vector of unknowns with restrictions on some components of $\mathrm{T}$ to take on integer values. MILP problems can be conveniently solved using branch-and-bound method [21] and can be generally represented as: 


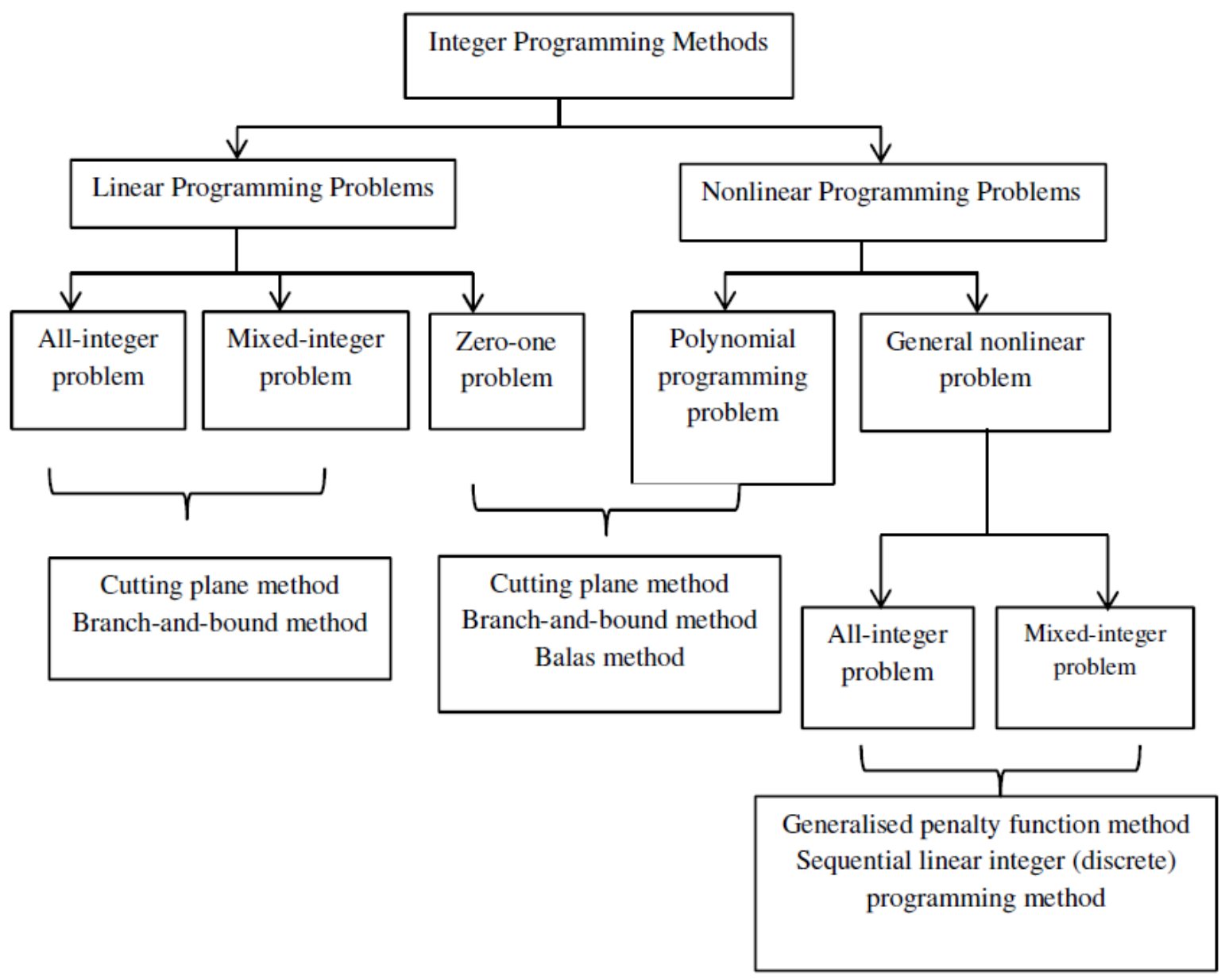

$$
\begin{gathered}
\operatorname{Min} C^{\mathrm{T}} X \\
\text { s.t. } \mathrm{Ax}=\mathrm{b} \\
\mathrm{x} \geq 0, \mathrm{x}_{1} \in \mathrm{Z} /, ; \forall \mathrm{i} \in \mathrm{I} .
\end{gathered}
$$

$\mathrm{CP}$ has attractive theoretical properties and has found applications in the development of efficient and reliable numerical algorithms. It unifies and generalises some common optimisation problems such as least square, quadratic programming and linear programming problems. A CP problem is of the general form:

$$
\min f(x)
$$

$$
\text { s.t. } g_{i}(x) \leq 0, i=1, \ldots . m
$$

Where functions $\mathrm{f}(\mathrm{x}), \mathrm{g} 1(\mathrm{x}) \ldots \ldots \ldots \ldots . . \mathrm{gm}(\mathrm{x}): \mathrm{IR} \rightarrow \mathrm{IR}$ are convex functions satisfying the condition:

$$
\begin{gathered}
\mathrm{f}(\propto \mathrm{x}+(1-\propto) \mathrm{y}) \leq \mathrm{f}(\mathrm{x})+(1-\propto) \mathrm{f}(\mathrm{y}) \\
\propto \in[0,1], \mathrm{x}, \mathrm{y} \in \mathrm{IR} .
\end{gathered}
$$

If $f(x)$ is a convex function then $-f(x)$ is a concave function. $\mathrm{CP}$ problems are commonly solved using the interior-point method. Convex and concave functions are illustrated in Figure.2:
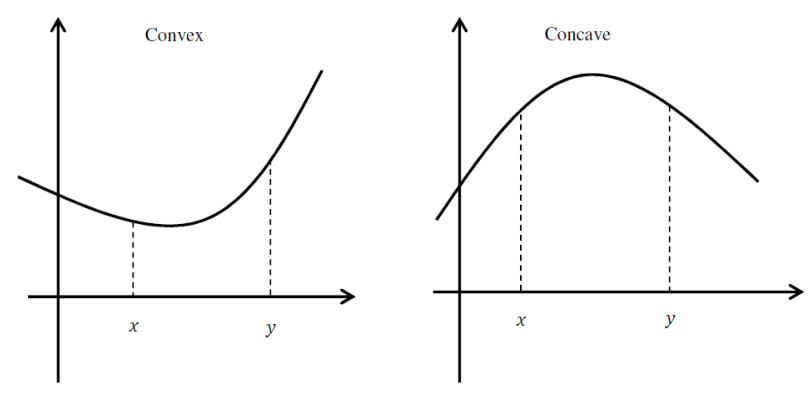

Figure 2: Illustration of convex and concave

$$
\text { minimize } \quad 1 / 2 \mathrm{X}^{\mathrm{T}} \mathrm{Qx}+\mathrm{c}^{\mathrm{T}} \mathrm{X}
$$$$
\text { subject to } \mathrm{Ax} \leq \mathrm{b} \text {. }
$$ 


\section{Hamrouni / Advances in Science, Technology and Engineering Systems Journal 5, 4, 99-104 (2020)}

Suppose an $\mathrm{n}$-dimensional vector is determined by $\mathrm{w}$, and $\mathrm{n} \mathrm{x}$ $\mathrm{n}$-dimensional real symmetric matrix is presented as $\mathrm{Q}, \mathrm{b}$ is an $\mathrm{m}$ dimensional real vector and n-dimensional real matrix.

Stochastic programming involves optimisation problems that have uncertain objective or constraint functions, which are characterised by a probability distribution on the variables.

Examples of mathematical programming methods found in DSM literature include linear/ integer/ binary/ mixed integer linear programming [22] [23], quadratic programming (QP), convex programming (CP), nonlinear programming (NLP) [24], stochastic programming (SP), heuristics and metaheuristic programming, and mixed integer nonlinear programming (MINLP).

Additional sensitivity analyses were carried out to study the robustness of the ESDS algorithm [25] [26], on PAR demand, dissatisfaction cost. First, the effect of battery capacity on consumer dissatisfaction was investigated. It was discovered that battery capacity has an indirect relationship to dissatisfaction of energy consumption. That is, the higher battery capacity value which can be acquired by consumers as iHES device, the less the consumer will be dissatisfied by appliance scheduling to an extent.

However, capacity of battery can never indefinitely increase; so, the diminishing law returns and set on savings point and then battery pay-back period. Simulation result of battery capacity assumed to be $7 \mathrm{kWh}$ [27]. Therefore, $4 \mathrm{kWh}$ and $10 \mathrm{kWh}$ battery capacities were simulated and the results obtained are presented in Figure.3.

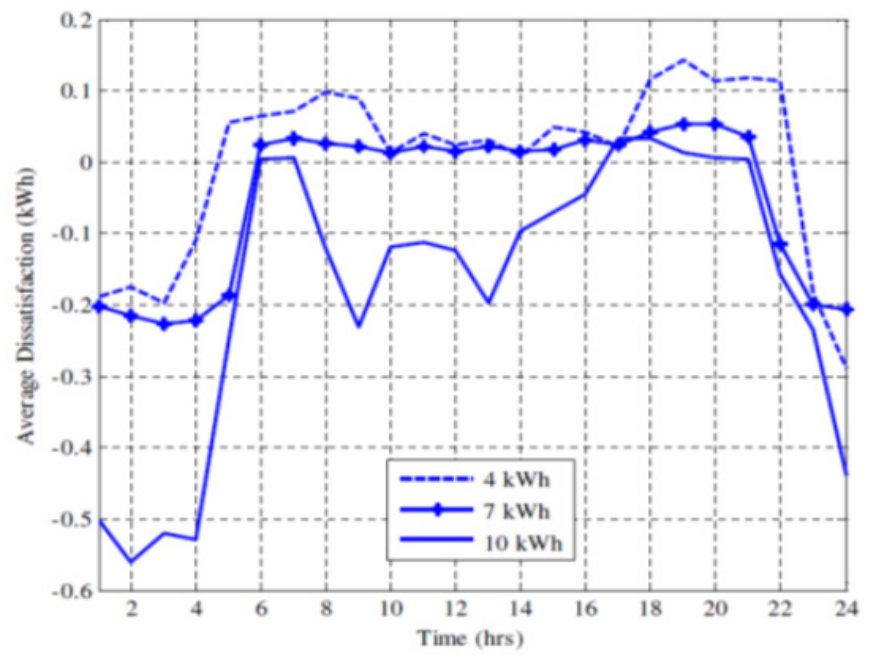

Figure 3: Relation between battery capacity and dissatisfaction coast

[28], [29], is effective in terms of robustness and convergence of algorithm. The performance of optimization techniques depends on user interactions and time scale.

The algorithm determines the energy value stored in the battery, it purchases from the grid and schedules demand to be met within the capacity of stored energy, at mentioned price. The results showed that peak period dissatisfaction, which is a common feature in DSM programs, and based on incorporation distributed energy storage system, it can be reduced. Consumer premises applying above constraints. The effect of DSM scheduling on dissatisfaction cost is shown in Figure.3.

\section{Conclusion}

Smart grid, Demand Side Management and mathematical optimisation techniques were reviewed in this work. Optimisation of energy consumption, distributed generation and storage can contribute towards mutually beneficial and satisfactory Demand Side Management Algorithms, which can be installed into consumer smart meters. These Demand Side Management Algorithms can be designed using any appropriate mathematical programming methods depending on the nature of their objective functions, variables and constraints. The DSM algorithms proposed in this paper are DMES, DOS-EUP.

\section{Conflict of Interest}

The author declares no conflict of interest.

\section{Acknowledgment}

The Authors would like to acknowledge the financial support of this work by Taif University- Khurma Univerity Collegue- Kidom of Saoudia Aribya.

\section{References}

[1] W. Kempton and A. Dhanju, "Electric vehicles with V2G: storage for largescale wind power", Windtech International, 2006.

[2] M. D. Galus, and G. Andersson, "Balancing Renewable Energy Sources using Vehicle to Grid Services controlled by MPC in a Metropolitan Area Distribution Network", in Proc.CIGRE ELECTRA, 2012.

[3] P. Mesaric and S. Krajar, "Home demand side management integrated with electric vehiclesand renewable energy sources," Energy and Buildings, 108, 1-9, 2015. Doi:10.1016/j.enbuild.2015.09.001

[4] M. D. Galus, M. G. Vayá, T. Krause, and G. Andersson, "The role of electric vehicles in the smart grid," Wiley Interscience Interdisciplinary Reviews of WIREs, Energy andEnvironment (WENE), 2, 4, 384-400, 2013. doi:10.1002/wene. 56

[5] W. Kempton and J. Tomic, "Vehicle-to-grid power fundamentals: calculating capacity and net revenue," Journal of Power Sources, 144, 1, 268-279, 2005. doi.org/10.1016/j.jpowsour.2004.12.025

[6] J. Suk-yee, Domestic electric vehicle market showing rapid growth, Business Korea/10126-governmental-support-domestic-electric-vehicle-marketshowing-rapid-growth, 2016.

[7] W. H. Fleming and R. W. Rishel, Deterministic and Stochastic Optimal Control, Springer, 1975 .

[8] A. K. Dixit, Optimisation in economic theory, Oxford, UK: Oxford University Press, 1990.

[9] K. Schmedders, Numerical optimisation methods in economics, The New Palgrave Dictionary of Economics, 2008.

[10] S. S. Rao, Engineering Optimisation Theory and Practice, John Wiley and Sons Inc, Hoboken, New Jersey, 4th Edition, 2009.

[11] V. F. Dem'yanov, V. N. Malozemov and D. Louvish, Introduction to Minimax, Keter House Jerusalem Ltd., 2014.

[12] M. Grant and S. Boyd, CVX: Matlab software for disciplined convex programming, CVX Research Inc., 2015.

[13] S. Boyd and L. Vandenberghe, Convex Optimisation, Cambridge University Press, New York, United States of America, 7th Edition,2009.

[14] G. B. Dantzig and M. N. Thapa, Linear programming 1: Introduction, Springer, 1997

[15] Y. Nesterov and A. Nemirovski, Interior-point polynomial algorithms convex programming, Studies in Applied Mathematics, SIAM, Philadelphia, 1994

[16] R. E. Gomory, "An Algorithm for the Mixed Integer Problem," Rand Report R.M. 25797, July 1960.

[17] J. Nocedal, S. Wright and J. Stephen, Numerical Optimisation, 2nd Edition, Springer- Verlag, Berlin, New York, 2006.

[18] A. H. Land and A. Doig, An Automatic Method of Solving Discrete Programming problems, Econometrica, 1960.

[19] A. Shapiro, D. Dentcheva and A. Ruszczynski, Lecture notes on stochastic programming - modeling and theory, Siam, 2009. 
[20] B. Daryanian, R. Bohn, and R. Tabors, "Optimal demand-side response to electricity spot prices for storage-type customers," IEEE Transactions on Power Systems, 4, 3, 897-903, 1989. Doi: 10.1109/59.32577

[21] D.S. Chen, R. G. Batson and Y. Dang, "Applied Integer Programming: Modeling and Solution," John Wiley and Sons, 2010.

S.P.Bradley, A.C.Hax and T.L.Magnanti, "Applied Mathematical Programming," Addison-Wesley, 1977.

[22] Z. Mao and X. Wang, "Efficient optimal and suboptimal radio resource allocation in ofdma system," IEEE Transactions on Wireless Communications, 7, 2, 440-445, 2008. Do1: 10.1109/TWC.060546

[23] R. E. Moore, Global optimisation to prescribed accuracy, Computers and Mathematics with Applications, Eldon Hensen, G.Willamwalster, 1991.

[24] D. Chen, X. Ziqi, L. Ximeng, Y. Yin, Y. Yang, G. Wenzhong, "Dual alSearch Artificial Bee Colony Algorithm for Engineering Optimization", IEEE Access, 7, 24571 -24584, 2019, Electronic ISSN: 2169-3536, INSPEC Accession Number: 18501113, Doi: 10.1109/ ACCESS.2019.2899743

[25] G. Cyrielle, B. Renaud, P. Marc, "Automatic Detection of Elevation Changes by Differential DSM Analysis: Application to Urban Areas", IEEE Journal of Selected Topics in Applied Earth Observations and Remote Sensing, 7, 10, 2014, : 4020 - 4037, Doi: 10.1109/JSTARS.2014.2300509

[26] C. Hamrouni, A. Bsissa, R. Hamza, N. Abdelkarim, "A new MEM-DOA proposal for DSM in a grid connected smart microgrid", Progress In Electromagnetics Research Symposium-Spring (PIERS), 22-25 May 2017. Doi: 10.1109/PIERS.2017.8262039

[27] W. Han; X. Sean Lu; M. Zhou; X. Shen; J. Wang; J. Xu,” Evaluation and Optimization Methodology for Efficient Power Plant Programs",IEEE Transactions on Systems, Man, and Cybernetics: Systems, IEEE Transactions on Systems, Man, and Cybernetics Systems, 50, 2, $707-716,2020$. Doi: 10.1109/TSMC.2017.2714198

[28] W. Lanneer, P. Tsiaflakis, J. Maes, M. Moonen," $\alpha$-Fair Dynamic Spectrum Management for QRD-Based Precoding With User Encoding Ordering in Downstream G.Fast Transmission", IEEE Transactions on Communications, 67, 4, 2939 - 2950, 2019. Doi: 10.1109/TCOMM.2018.2890237.

[29] D. Li. C. Wei, S. Hongiian, H.P. Vincent,"Multi-objective Optimization for Demand Side Management Program in Smart Grid", IEEE Transactions on Industrial Informatics, 14,4, $1482-\quad 1490,2018$. Doi: 10.1109/TII.2017.2776104. 